(1)

CrossMark

\section{The clinical dynamics of 18 cases of COVID-19 outside of Wuhan, China}

\author{
To the Editor:
}

Since an outbreak of coronavirus disease 2019 (COVID-19) in Wuhan, China, and related regions of the Hubei province, an increasing number of exported cases have been confirmed in other provinces of China and in multiple countries around the world, with substantial morbidity and mortality [1-4]. The World Health Organization (WHO) has declared a public health emergency of international concern considering rapid increases in numbers of confirmed cases in China and additional countries. As of 22 February 2020, a total of 12938 patients had been confirmed outside of Wuhan and related regions of the Hubei province of China [1]. However, there is limited information about COVID-19 outside of Wuhan [5], and no study has reported the time to real-time PCR conversion and radiological changes after treatment.

We recruited all patients who were diagnosed as having COVID-19 from 21 January to 5 February 2020, at the First Affiliated Hospital of Zhengzhou University in Zhengzhou, China. The final date of follow-up was 7 February 2020. Throat-swab specimens were collected and sent to the Center for Disease Control and Prevention of Henan province (Zhengzhou, China) and severe acute respiratory syndrome coronavirus 2 (SARS-CoV-2) was detected using real-time PCR. Chest computed tomography (CT) was performed on admission and at the time of real-time PCR conversion. Data were collected from electronic medical records, with epidemiological and symptom data confirmed by directly communicating with patients or their family members (interviews conducted by L. Wang and L-L. Lou). The date of symptom onset referred to the day when the symptom was noted. Time to real-time PCR conversion was calculated from the date of symptom onset to the day when SARS-CoV-2 was undetectable from two consecutive throat-swab specimens. Patients could be discharged from hospital when they had real-time PCR conversion and no fever for $\geqslant 3$ days according to the interim guidance from Chinese National Heath Commission on clinical management of COVID-19 [6]. The study was approved by the Ethics Committee of the First Affiliated Hospital of Zhengzhou University, with a waiver of written informed consent.

18 patients with COVID-19 were included, with one familial cluster of infection. Of these patients, $10(55.6 \%)$ were male, with a median age of 39 years (interquartile range 29-55). For the familial cluster of infection (including two children aged 7 and 9 years), five of the six family members had tested positive for SARS-CoV-2 and the remaining family member was highly suspected due to bilateral ground-glass opacities on chest CT, although real-time PCR was negative. 13 (72.2\%) had a history of visiting Wuhan. $12(66.7 \%)$ did not have underlying conditions. Six $(33.3 \%)$ had chronic comorbidities, including three (16.7\%) with cardiovascular disease, five $(27.8 \%)$ with hypertension, three $(16.7 \%)$ with diabetes, two (11.1\%) with stroke and one (5.6\%) with a malignant tumour.

On admission, most patients presented as fever (94.4\%) or cough (55.6\%). Other symptoms included shortness of breath $(22.2 \%)$, haemoptysis $(5.6 \%)$, muscle ache $(11.1 \%)$, headache $(5.6 \%)$, sore throat (5.6\%), diarrhoea (16.7\%), nausea and vomiting (5.6\%). One patient had acute lung injury and was admitted to the intensive care unit (ICU).

From the laboratory results (data available for 16 patients) and chest CT, it was noted that white blood cells were below the normal range in three (18.8\%) patients and above the normal range in one $(6.3 \%)$ patient. Three (18.8\%) patients had neutrophils above the normal range. Lymphopenia was found in eight (50\%) patients. Four (25\%) patients had abnormal liver function, with alanine aminotransferase and aspartate aminotransferase above the normal range. Regarding infection markers, C-reactive protein

@ERSpublications

SARS-CoV-2 can be transmitted from person to person, with infection ranging from mild disease to severe pneumonia and radiological abnormalities on chest CT for most patients, improving after RT-PCR conversion http://bit.ly/2TiMSck

Cite this article as: Wang L, Gao Y-h, Lou L-L, et al. The clinical dynamics of 18 cases of COVID-19 outside of Wuhan, China. Eur Respir J 2020; 55: 2000398 [https://doi.org/10.1183/13993003.00398-2020]. 

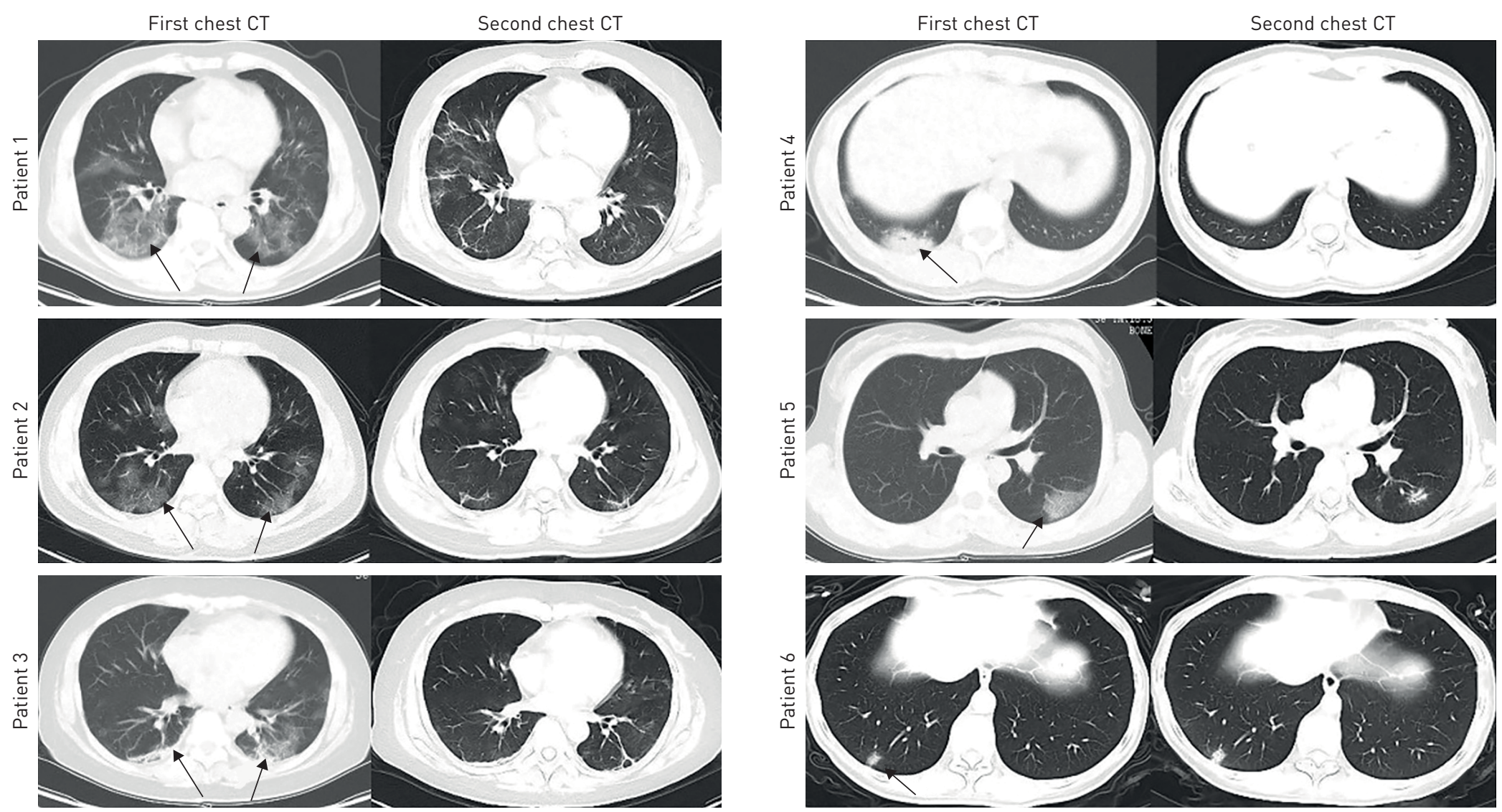

FIGURE 1 Representative chest computed tomography (CT) images before admission and at the time of real-time PCR conversion in six discharged patients. Patient 1: first chest CT obtained on 23 January 2020 showed ground-glass opacities in both lung larrowsl. Second CT on 1 February 2020 showed healing of the ground-glass opacities. Patient 2: first chest CT obtained on 22 January 2020 showed ground-glass opacities in both lungs larrows). Second CT on 4 February 2020 showed resolution of bilateral ground-glass opacities Patient 3: first chest CT obtained on 22 January 2020 showed ground-glass opacities with consolidations in both lungs larrows). Second CT on 4 February 2020 showed the resolution of bilateral ground chest CT obtained on 22 January 2020 showed ground-glass opacities with consolidations in both lungs larrows). Second CT on 4 February 2020 showed the resolution of bilateral ground glass and consolidations. Patient 4: first chest CT obtained on 21 January 2020 showed consolidations with mild ground-glass opacities in the right lung (arrow). Second CT on 4 February showed resolution of left ground-glass opacities. Patient 6: first chest CT obtained on 23 January 2020 showed mild consolidation in the left lung larrow). Second CT on 4 February 2020 showed that the consolidation remained. 
$>5.0 \mathrm{mg} \cdot \mathrm{L}^{-1}$ was very common (noted in 12 (75\%) patients). In contrast, four (25\%) patients had procalcitonin above the normal range $\left(>0.046 \mathrm{ng} \cdot \mathrm{mL}^{-1}\right)$. Of 18 patients who had chest CT, 15 patients had pneumonia, with $11(73.3 \%)$ showing as bilateral lung involvement and the remaining three (16.7\%) with normal chest CT. Of the 15 patients with pneumonia on chest CT, all had ground-glass opacities and seven $(46.6 \%)$ also had consolidation.

By 7 February 2020, six (33.3\%) patients had been discharged and all the other patients were still in the hospital. $13(72.2 \%)$ patients had fully symptomatic remission and one had developed to severe respiratory failure and been transferred to the ICU. One patient who was referred to the ICU on admission was then transferred to a general isolated ward on the 8th day of admission.

The median time from symptom onset to admission was 7 days (range 2-15 days). Of the 13 fully symptomatic remission patients, the median time from illness onset to symptom remission was 10 days (range 3-15 days). Of the six discharged patients, the median time of real-time PCR conversion was 19.5 days (range 17-24 days) and the interval between the two chest CT scans was 12.5 days (range 10-15 days). Five (83.3\%) had significant radiological improvement at the time of real-time PCR conversion and the remaining patient had no change on chest CT image (figure 1).

Our results firstly show that the time to real-time PCR conversion was 19.5 days for COVID-19 and most patients had significant radiological improvements after real-time PCR conversion. Consistent with previous studies, most patients presented with fever, dry cough and shortness of breath [2-4]. The patterns of radiological appearance were bilateral ground-glass opacities with or without consolidations [7]. All these features resemble SARS-CoV and Middle East respiratory syndrome (MERS)-CoV [8, 9]. However, patients with COVID-19 can present with mild disease or even no symptoms, and no lung abnormalities or severe pneumonia on chest CT images. We reported a family cluster infection and in some confirmed cases, there were no comorbidities or no history of visiting Wuhan, suggesting human-to-human transmission occurred outside of Wuhan and regardless of age or underlying conditions [10-12]. Compared with patients in Wuhan, the manifestations in patients in the Henan province were relatively mild [2-4], which was consistent with 13 case series of COVID-19 in Beijing, China [5]. Notably, transmission in an asymptomatic carrier has been reported [12], which might pose more threat than symptomatic patients to epidemic prevention and control.

The median time from symptom onset to real-time PCR conversion in patients with COVID-19 was 19.5 days, which seems to be shorter than patients infected with SARS-CoV or MERS-CoV [13, 14]. Previous studies in SARS-CoV or MERS-CoV showed that viral RNA could be detected in clinical specimens in patients $>30$ days after symptom onset $[13,14]$. It is worth mentioning that we only used the throat-swab specimen to detect the viral RNA. Evidence has shown that SARS-CoV-2, SARS-CoV and MERS-CoV RNA can be detected in both the upper and lower respiratory tract samples, even in blood, stool and urine specimens $[2,13,14]$. Because the lower respiratory tract was thought to be the main target of coronavirus and the viral RNA level is often higher in the lower respiratory tract specimen [15], we should be cognisant of the potential for prolonged viral shedding in the lower respiratory tract. Further data on ascertaining the duration of infectivity of COVID-19 by monitoring real-time PCR status in differing levels of severity using multiple clinical specimens are merited. Nevertheless, patients with COVID-19 should continue to follow hygiene measures after discharge from hospital, including wearing a surgical mask, disinfection of food utensils and toilet disinfection. For patients with mild pneumonia, radiological abnormalities were significantly improved in most patients after real-time PCR conversion. However, the consistency between real-time PCR conversion and the resolution of lung abnormalities requires further study in patients with critical illness.

In summary, COVID-19 can present a variety of manifestations ranging from no symptoms or mild disease to resolved or severe pneumonia. Real-time PCR conversion was compatible with improvement of radiological abnormalities. Large studies are urgently needed to explore the duration of infectivity and related factors of COVID-19.

Lei Wang ${ }^{1,2}$, Yong-hua Gao ${ }^{1,2}$, Li-Li Lou ${ }^{1}$ and Guo-Jun Zhang $^{1}$

${ }^{1}$ Dept of Respiratory and Critical Care Medicine, The First Affiliated Hospital of Zhengzhou University, Zhengzhou, China. ${ }^{2}$ Both authors contributed equally as first authors.

Correspondence: Yong-hua Gao, Dept of Respiratory and Critical Care Medicine, The First Affiliated Hospital of Zhengzhou University, Zhengzhou, Henan, China. E-mail: gaoyonghuahust@163.com

Received: 09 Feb 2020 | Accepted after revision: 25 Feb 2020

Author contributions: Y-H. Gao and L. Wang designed the project. L. Wang, L-L. Lou and G-J. Zhang carried out the data collection. Y-H. Gao analysed the data and prepared the figures. Y-H. Gao and L. Wang drafted the manuscript. 
All the authors have revised the manuscript critically, approved the version submitted for publication and have agreed to be accountable for all aspects of the work.

Conflict of interest: None declared.

\section{References}

1 World Health Organization. Coronavirus disease 2019 (COVID-19): situation report-33. https://www.who.int/docs/ default-source/coronaviruse/situation-reports/20200222-sitrep-33-covid-19.pdf Date last accessed: 22 February 2020.

2 Huang C, Wang Y, Li X, et al. Clinical features of patients infected with 2019 novel coronavirus in Wuhan, China. Lancet 2020; 395: 497-506.

3 Chen N, Zhou M, Dong X, et al. Epidemiological and clinical characteristics of 99 cases of 2019 novel coronavirus pneumonia in Wuhan, China: a descriptive study. Lancet 2020; 395: 507-513.

4 Wang $\mathrm{D}, \mathrm{Hu} \mathrm{B}, \mathrm{Hu} \mathrm{C}$, et al. Clinical characteristics of 138 hospitalized patients with 2019 novel coronavirus-infected pneumonia in Wuhan, China. JAMA 2020; in press [doi:10.1001/jama.2020.1585].

5 Chang D, Lin M, Wei L, et al. Epidemiologic and clinical characteristics of novel coronavirus infections involving 13 patients outside Wuhan, China. JAMA 2020; in press [doi:10.1001/jama.2020.1623].

6 Chinese Municipal Health Commission. The management of 2019 novel coronavirus infected pneumonia: interim guidance. http://www.nhc.gov.cn/yzygj/s7653p/202001/f492c9153ea9437bb587ce2ffcbeelfa/files/39e7578d85964dbe 81117736dd789d8f.pdf

7 Song F, Shi N, Shan F, et al. Emerging coronavirus 2019-nCoV pneumonia. Radiology 2020: 200274.

8 Lee N, Hui D, Wu A, et al. A major outbreak of severe acute respiratory syndrome in Hong Kong. $N$ Engl J Med 2003; 348: 1986-1994.

9 Assiri A, Al-Tawfiq JA, Al-Rabeeah AA, et al. Epidemiological, demographic, and clinical characteristics of 47 cases of Middle East respiratory syndrome coronavirus disease from Saudi Arabia: a descriptive study. Lancet Infect Dis 2013; 13: 752-761.

10 Chan JF, Yuan S, Kok KH, et al. A familial cluster of pneumonia associated with the 2019 novel coronavirus indicating person-to-person transmission: a study of a family cluster. Lancet 2020; 395: 514-523.

$11 \mathrm{Li} \mathrm{Q}$, Guan $\mathrm{X}, \mathrm{Wu}$ P, et al. Early transmission dynamics in Wuhan, China, of novel coronavirus-infected pneumonia. N Engl J Med 2020; 382: 1199-1207.

12 Bai Y, Yao L, Wei T, et al. Presumed asymptomatic carrier transmission of COVID-19. JAMA 2020; in press [doi:10.1001/jama.2020.2565].

13 Bin SY, Heo JY, Song MS, et al. Environmental contamination and viral shedding in MERS patients during MERS-CoV outbreak in South Korea. Clin Infect Dis 2016; 62: 755-760.

14 Chu CM, Leung WS, Cheng VC, et al. Duration of RT-PCR positivity in severe acute respiratory syndrome. Eur Respir J 2005; 25: 12-14.

15 Killerby ME, Biggs HM, Midgley CM, et al. Middle east respiratory syndrome coronavirus transmission. Emerg Infect Dis 2020; 26: 191-198. 